\title{
Demonstration of Participation Networks in Urban Transport Policy of Public and Private Sectors through Social Media: The Case of Bike-Sharing Pricing Strategy in China
}

\author{
Qian Ye $\mathbb{D}^{1},{ }^{1}$ Xiaohong Chen, ${ }^{1}$ Hua Zhang $\mathbb{D}^{2},{ }^{2}$ Junjie Cai, ${ }^{3,4}$ and Kaan Ozbay ${ }^{3}$ \\ ${ }^{1}$ Key Laboratory of Road and Traffic Engineering of the Ministry of Education, School of Transportation Engineering, \\ Tongji University, Shanghai 201804, China \\ ${ }^{2}$ National Maglev Transportation Engineering R\&D Center, Tongji University, Shanghai 201804, China \\ ${ }^{3}$ C2SMART Center, Department of Civil and Urban Engineering, Tandon School of Engineering, New York University, \\ New York, NY 11201, USA \\ ${ }^{4}$ Baidu Inc., Beijing 100085, China \\ Correspondence should be addressed to Hua Zhang; xiaohai_hua@tongji.edu.cn
}

Received 8 May 2020; Revised 9 June 2020; Accepted 15 April 2021; Published 27 April 2021

Academic Editor: Jaeyoung Lee

Copyright (c) 2021 Qian Ye et al. This is an open access article distributed under the Creative Commons Attribution License, which permits unrestricted use, distribution, and reproduction in any medium, provided the original work is properly cited.

Social media has become a valuable platform that enables public and private stakeholders to participate and interact in various transport policies. Using a network-based perspective and a case study of bike-sharing pricing strategies in China, this paper aims to quantitatively characterize the pattern and structure of multi-stakeholders engagement networks. Furthermore, this paper also empirically examines the confirmation bias that might exist among participants. Dataset on retweets from the Chinese TwitterSina Weibo is collected. Results reveal two types of important actors with unequal roles in terms of information diffusion: the "network root" and the "network bridge." The former is mainly comprised of organizations and influential individuals who dominate message sharing, whereas the latter is comprised of the general public with various occupational backgrounds who control the efficiency and the scope of information spreading. The result also reveals a hierarchical structure in both networks and a community gathering like-minded individuals. The empirical result also demonstrates the existence of echo chambers in the transport participation network of governments and enterprises. Most echo chambers operate such that organizations or influential individuals amplify the views of the general public with more critical viewpoints. These findings of this study can assist transport stakeholders in crafting more sustainable strategies based on the understanding of uneven patterns in online public participation. Furthermore, this study sheds insights on how social media could be used to facilitate the collection of diverse people's opinions and the evaluation of multi-stakeholder engagement for major transport issues.

\section{Introduction}

Making appropriate policies or strategies is essential to the sustainable development of urban transport. Traditionally, transport policy-making processes are characterized by a top-down approach that the public seldom has access to, for example, hearings, expert consultants, or publications. It causes ineffective communication between citizens, private sectors, and administrative agencies as well as limited transparent governance [1]. In recent years, with the emerging of new mobility patterns and services, transport governance may be undertaken by multiple stakeholders who have various interests, usually requiring interdisciplinary and multiagent cooperation [2]. Consequently, it highlights the differences in urban transport between the concept of government-driven sharing and that of collaboration among multi-stakeholders, i.e., market regulators, service providers, and transport system users.

Social media has been widely recognized as being able to facilitate the engagement of experts, private sectors, civic organizations, and the public as well as being able to create large and diffused networks of relationships $[3,4]$. Due to the 
potential role of social media in extracting peoples' real thoughts and addressing the complexity of stakeholder relations in the transport field, scholars have explored its applications on both bottom-up and top-down engagement in urban transport [5]. However, this research remains in the infant stage.

In recent years, more and more private stakeholders (i.e., platform operators, service providers) have offered personal and urban level mobility services and thus a method for opinion collection that can be significant to business promotion strategies. As such, the structure and characteristics of public participation might be different in public and private decision-making processes in the transport field. However, few scholars have investigated the policy-making participation network from a comparative perspective.

Most recently, a phenomenon of confirmation bias has arisen from concerns about decision-making or information processing: that is, the tendency to search for, interpret, and recall information in a way that is consistent with existing beliefs [6]. In social media, it is defined as an echo chamber or filter bubbles [7]. The presence of echo chambers leads to information barriers around people and, thus, causes difficulties in sharing and understanding diverse viewpoints and even reaching a solution. As such, it is necessary to confirm the existence and the degree of confirmation bias on the collected social media data before forming a conclusion on transport policy issues. However, little evidence has concerned echo chambers in transport policies and their influence on the public and private sectors.

For those reasons, we considered it important to present and analyze the pattern of participation networks in transport policy-making led by governments (GCPN) and enterprises (ECPN). This study also aims to empirically examine the existence of confirmation bias among participants in terms of personal attitude diffusion. Two topics on bike-sharing pricing strategies resulting from government regulations and business strategies are empirically studied, by collecting the retweet data from the Chinese Twitter-Sina Weibo. The specific objectives are (i) to illustrate the pattern and characteristics of the citizen involvement in transport policies by public and private sectors, (ii) to simplify the structure of participation networks and identify influential actors/groups with key roles in information dissemination, and (iii) to empirically examine the degree of confirmation bias in transport policy participation networks. The results can help multiple stakeholders to improve transport policies from a perspective of the nature of online public engagement. Furthermore, results provide insights into the design of evidence-driven policy research to avoid the potential bias when using social media as a platform to gather opinions and needs of people from different backgrounds concerning transport issues.

\section{Literature Review}

2.1. Online Public Participation in the Transport PolicyMaking. Online platforms (e.g., social media, blogs, and websites) have become a vital channel for public involvement that may be supplementary to traditional or face-to- face engagement opportunities [8]. Increasing theoretical efforts and implementations have been devoted to the potential of social media in understanding the complex interactions between transport stakeholders and the public in the transport domain $[9,10]$. For example, transport policymakers and providers in the USA, like MTA Transit and Metro-North Railroad in New York City, and DOTs in Alabama, California, West Virginia, etc., use Facebook and Twitter to inform travel information or specific events as well as to connect the feedback from the residents, so that to facilitate project improvements and misinformation corrections $[8,11]$. Furthermore, with the support of web-based tools/toolkits and text mining techniques [12], ridesharing agencies can optimize ridership, timeliness, efficiency, and safety and also reveal the level of satisfaction with ridesharing services by monitoring social media [13, 14]. Another example is the adjustment of the No. 55 bus route in Shanghai. The transport agencies and local magazines disseminated the message about the abolition of the bus route on Weibo. Since a large number of citizens commented on this message and expressed their nostalgia for the bus route, the local government was pushed to interact with citizens and completely change the initial plan [15].

To date, many methodologies like network science are developed to demonstrate the complex participation networks involving multiple stakeholders [16]. Social Network Analysis (SNA) is a method proposed by sociologists to measure the positions and structural characteristics of relationships between social actors $[17,18]$. The main research topics of SNA in the transport field are summarized into three categories: megaproject collaboration and management [19, 20], multimodal transport network analysis $[21,22]$, and participatory decision-making. Of very few studies using SNA on transport policy issues, Dörry and Decoville study the situated multilevel governance structure of cross-border public transport provision by taking the respective cross-border policy network in the Luxembourg metropolitan region as a case [23]. Sun et al. use the SNA method to refine multi-actor multicriteria analytical results and further evaluate various low-carbon transport policies [24].

According to the study conducted by Weiss et al. and Mills et al., SNA can highlight which actors are important for influencing policies or actions, or for facilitating information and knowledge transmission [25, 26]. Therefore, this study applies the SNA method to simplify the structure of participation networks in terms of public and private policymaking.

2.2. Confirmation Bias on Social Media and Real-World Policy Networks. Confirmation bias is a psychological phenomenon where humans tend to seek information adhering to their preexisting opinion [27], which has become a widely debated issue at both the scientific and political levels [28]. Recent studies have shown that information bias is dominant in the social media environment [29, 30], where individuals are surrounded by people whose viewpoint agrees with their own, also known as echo chambers or filter 
bubbles [7]. Major social media like Facebook and Twitter presents clear-cut echo chambers on controversial topics [31]. This configuration could cause the spreading of bias knowledge [32, 33], hamper the democratic deliberative process by altering the way facts are perceived [34], and even lead to a loss of common understanding [35]. Several studies develop the theoretical definitions and mechanisms of echo chambers [31]. For instance, Jasny, Waggle, and Fisher propose a more formal operationalization of echo chamber components, which comprises two processes of an "echo" and "chambers" and at least three types of actors-speakers, receivers, and mediating actors [36]. Cinelli et al. introduce a definition and method of echo chambers based on the coexistence of two main ingredients: opinion polarization concerning controversial topics and homophilic interactions between users [31].

From the policy network perspective, echo chambers are questioned to affect evidence-based policy-making as they can hinder policy learning and consensus building [28]. It has been demonstrated that organizations and individuals tend to obtain information from those with similar beliefs to theirs while ignoring information from sources that challenge their beliefs [36, 37]. Recent studies also provide empirical evidence of the effects of echo chambers on policy networks using survey data. For example, Jasny et al. compare two datasets on the US climate politics at two points in time and find that echo chambers continue to play an important role in the information sharing among policy elites working on climate issues [38]. Wagner and YläAnttila find that echo chambers are present in the relationship between the information-seeking behaviors of policy actors in Irish policy networks; also, they reveal that policy forums could help alleviate the effects of echo chambers if organizations with different views were to participate and to use the opportunities that forums provide to learn from those outside their networks [28].

Due to the complexity of disciplinary issues emerging in shared transport and the essentiality of information sharing among multiple stakeholders, the phenomenon of echo chambers is potentially influencing the policy-making in urban transport. Therefore, there is a need to test the existence of echo chambers in public and private policymaking and further to emphasize its applicability and effectiveness in the analysis of various transport policies.

\section{Background and Research Methods}

3.1. Case Information on Bike-Sharing Pricing Policies in China. The dockless bike-sharing service was introduced to China since the establishment of the Ofo platform in 2014. The system consists of government regulators, platform operators, bike associations, and bike-sharing users [39]. The number of bike-sharing users increased significantly within the first two years. However, due to the lack of sustainable business strategies and management, leading bike-sharing enterprises like Ofo have faced serious financial problems since the end of 2018. Millions of riders who placed deposits demanded their bicycle refunds, causing widespread concerns and debates among the public and government authorities on deposit-related issues of emerging travel services in China [40].

In response, the Ministry of Transport (MOT) in China formally has included the deposit issue in government supervision and published tighter controls soon. In March 2019, an administrative measure of user deposit in emerging transport was issued to gather advice from the public, followed by the final version of the implementation guidance that was released in May 2019 [41]. This document specifies the behaviors of service providers including the schedule of refunding user deposits and the way of deposit management. Soon after, several bike-sharing enterprises including Bluegogo, Mobike, and Hello announced their pricing increase strategies. More and more enterprises also employed a higher level of bike-sharing fares in the next months. The outline of bike-sharing pricing strategies in China is shown in Figure 1.

Therefore, this study chooses these two topics on Weibo to understand the citizen participation in public and private decision-making, corresponding to the fact that "Chinese governments have issued an administrative measure of user deposits in emerging transport services" and "many enterprises have launched the price increase in bike-sharing services."

3.2. Research Methods. The analytical framework of this study includes the collection of retweet data on Weibo, SNAbased measurement, and echo chamber examination, which is depicted in Figure 2.

3.2.1. Data Collection. A data set of Weibo retweets on the abovementioned two topics was collected on July 8, 2019. It consists of two sections: messages/retweets and information of participated users. A snowball sampling method was used to complete the sample of participants and their messages in this study. This data collection method has been employed in the analysis of social networks and social dynamics [42]. For each topic, the initial samples were top50 most-read messages on Weibo (called the initial messages). Then, messages forwarded from the initial messages by users were picked (called the first layer of retweets). Similarly, retweets of deeper layers were further searched until no new retweets were founded. The data set of participant information was gathered from their homepage on Weibo addressed by their usernames shown in the retweet records. Available features such as job, education, and identity verification were extracted. The verification setting on Weibo enables businesses, celebrities, etc. to show their credibility and trust, generally correlated with their numbers of followers and influence. The participants were correspondingly divided into three categories: influential individuals, organizations/companies, and the general public. Finally, a total of 2335 and 2845 participants who published 2469 and 2938 Weibo messages related to two topics were included in this study.

The next step was to reconstruct the forwarding/forwarded relationship among participants by parsing the content of retweets. Through identifying unique user IDs 


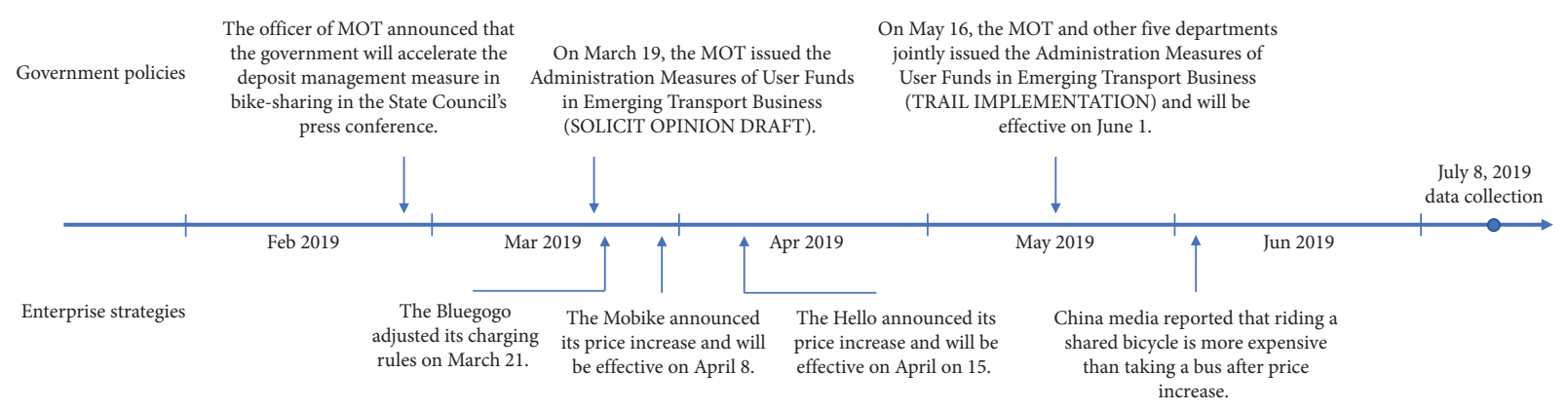

FIgURE 1: Overview of deposit-related policies of bike-sharing in China.

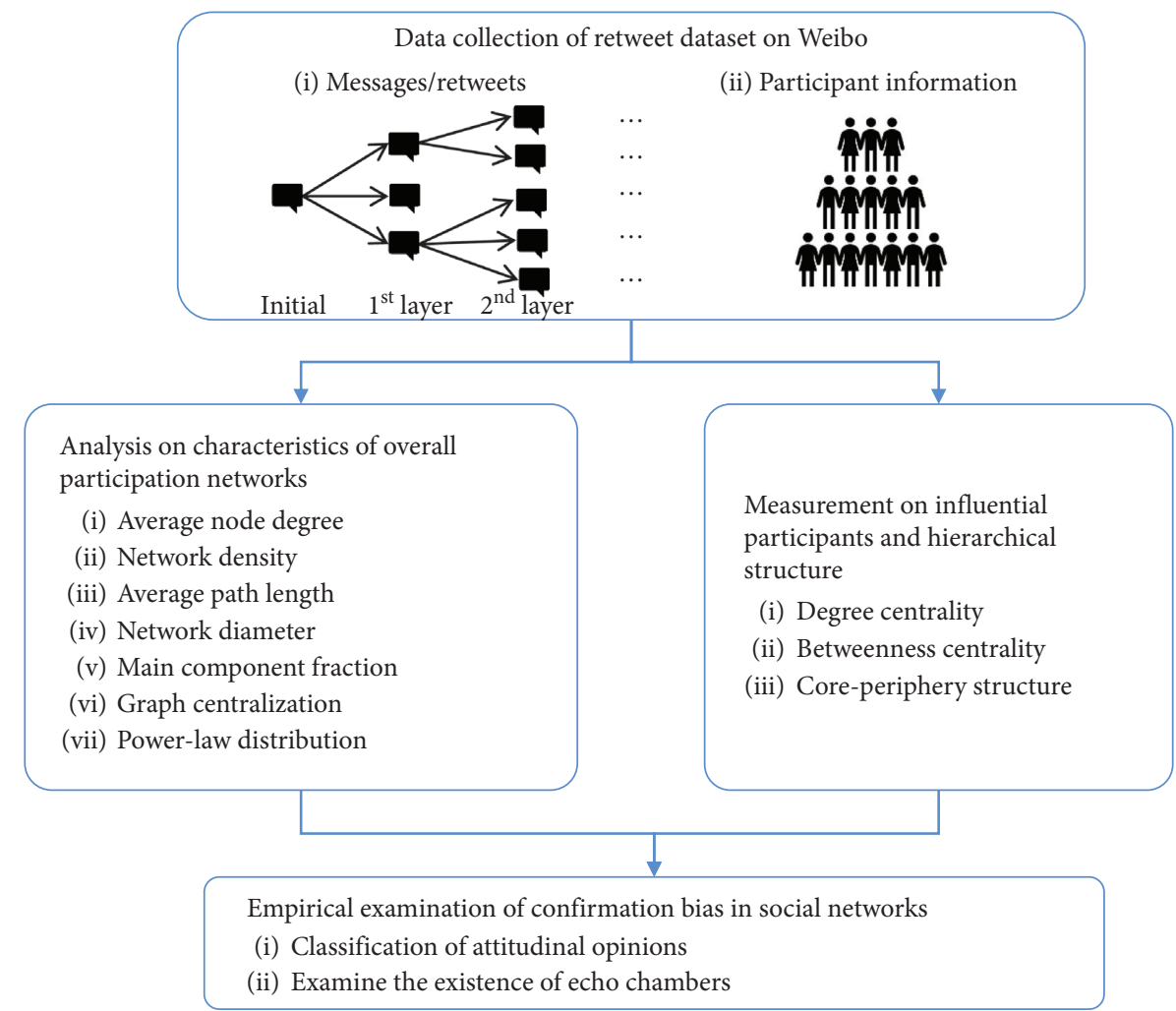

FIGURE 2: Research analysis framework in this study.

and the retweet identifier (“//@” on Weibo) in the message dataset, direct/indirect interactions and corresponding information flow started from the initial participants were obtained. To map and analyze the data sets, an adjacency matrix, a square matrix used to represent a finite graph, was used to represent the relational data among the pairs of vertices (participants) [43]. The number of each cell $r_{i, j}$ in the matrices was denoted as 1 for two participants (i.e., $i$ in the row and $j$ the column) who were directly linked in the forwarding/forwarded structure and denoted as 0 otherwise. A rule was applied here: that is, simplifying the multiple forwarding/forwarded between the same pair of users into one. Thus, the value of $r_{i, j}$ was binary, in which edges among participants were dichotomized as 1 or 0 .
3.2.2. Network Classification and Measurement. Given this unweighted directed graph, the network was formalized as a collection of participants as nodes and their forwarding/ forwarded relationships as edges using the SNA method. For example, there was a directed edge from actor A to actor B when B forwarded a message from A, which is consistent with the direction of information flows within networks. As this study focuses on comparing the participation networks of public and private sectors, datasets were further classified into two examined networks: citizen participation network led by governments (represent as GCPN) and enterprises (represent as ECPN). As such, a total of 2390 edges (nonzero cells $r_{i, j}$ ) in GCPN and 2873 edges in ECPN were analyzed. Descriptive analysis characterizes both the overall structure 
and the individual performance of network nodes. The indicators used are as follows [44]:

(i) Average node degree, which refers to the average number of edges per node in networks. It includes in- degree and out-degree which indicate the number of forwarded messages to and from each participant.

(ii) Network density, which measures the tightness of nodes that are calculated as the proportion of edges that are exiting in the network to the largest number of possible edges. Its values vary between 0 and 1 . The greater the density of networks, the more the contacts among participants.

(iii) Average path length, which refers to the average number of steps along the shortest paths for all possible pairs of nodes. It measures information efficiency on networks.

(iv) Network diameter, which refers to the shortest distance between the two most distant nodes in networks. It determines an upper bound of the distance between any pairs of participants who can exchange information.

(v) Main component fraction, which refers to the proportion of main components (or giant components). It is a node group with the largest number of interconnected nodes that emerged in networks.

(vi) Graph centralization, which refers to the degree to which the centrality of the most central node exceeds the centrality of all other nodes in overall networks. It varies between 0 and 1 , which quantifies the variation of individual nodes' importance and the distribution of node positions in networks.

(vii) Power-law distribution, which refers to the cumulative probability of nodes whose degree is larger than or equal to $k$ (represented as $D(k)$ ), to assess the unevenness of specific network indicators (e.g., out-degree). The distribution is formulated as follows [45], where $\alpha$ is the law's exponent and $C$ is a constant:

$$
\begin{aligned}
D(k) & =C \cdot k^{-\alpha}, \\
\ln D(k) & =\ln C-\alpha \ln k .
\end{aligned}
$$

Concerning the different roles of individual nodes and the hierarchical structure in each participation network, indicators of degree centrality and betweenness centrality, as well as k-core decomposition, were selected to identify influential participants/groups in terms of information dissemination. Degree centrality (DC) refers to the number of edges that a node has, which is a primary measure of the importance of nodes [46]. In a forwarding/forwarded network, a relatively high out-degree centrality indicates that the corresponding actor has more channels of information exchanges with other participants and is thus at a more central position in the entire network (i.e., "network root"). Betweenness centrality (BC) is another measure to capture essential nodes based on the shortest paths. It is calculated as the number of these shortest paths that pass through target nodes [47]. In terms of participation networks, a specific person with a high $\mathrm{BC}$ means that they played a significant role (e.g., "network bridge") in allowing information to travel from one part to another in the entire network.

The $k$-core decomposition is an alternative degree-based method to conduct hierarchical structure analysis, graph visualization, and community detection in complex networks [48]. The $k$-core indicates the maximal subgraph in which every node is adjacent to at least $k$ nodes. An integer index, $k_{s}$, is assigned to each node to represent its location in networks; the largest value is denoted as $k_{\max }$ (called $k$-core index). Each layer that contains nodes of degree $k$ is called $k$-shell. In this sample, participants with low or high $k_{s}$ are correspondingly located at the periphery or the core, also indicating the importance of nodes in the entire network. It should be noted that nodes with a high degree may or may not have a high $k_{s}$.

3.2.3. Echo Chamber Identification Algorithm. This study employs an operationalization of the components of echo chambers proposed by Jasny, Waggle, and Fisher [36]. Given the network formalism in Section 3.2.1 (represented in Figure 3(a)), two characteristics of echo chambers in participation networks are specified. An "echo" occurs when two participants share the same opinion on the policy. The attribute is indicated as the shading of the nodes in Figure 3(b). A "chamber" is formed when the information from the same source eventually reaches the same target via multiple different paths. For example, a transitive triad is the smallest example of a "chamber" where messages pass from participant $A$ to participant $C$ through a directed edge, but also indirectly through participant $\mathrm{B}$ as $\mathrm{B}$ forwards the message from $\mathrm{A}$ and then $\mathrm{C}$ forwards the message to $\mathrm{B}$ (see Figure 3(c)).

Thus, echo chambers can be demonstrated once the chamber is configured around participants holding the same opinions (see Figure 3(d)).

Considering the feature that retweets are more likely to be passed from a few network elites to a large number of users, this study developed a more effective identification algorithm to find echo chambers quickly from online participation networks.

(i) Step 1: search participants that forward information from multiple sources, for example, find out $\mathrm{C}$ who receives messages from at least two sources in Figure 3

(ii) Step 2: select the structure where connections between any two sources exist, that is, to find out a like-shaped transitive triad

(iii) Step 3: test the uniqueness of participants in the structure, and then find out the transitive trail that has three distinct participants - a "chamber"

(iv) Step 4: determine the presence of an "echo chamber" by checking whether the participants in chambers hold the same viewpoints 


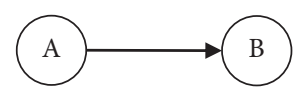

$\mathrm{B}$ forwards messages from A, i.e., information is transmitted from $\mathrm{A}$ to $\mathrm{B}$.

(a)

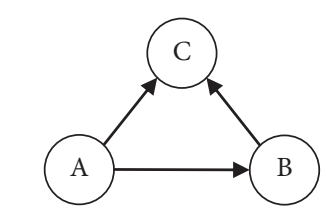

$A$ and $B$ agree so A's information echoes B's understanding.

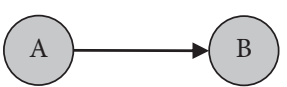

Transitive triad such that $\mathrm{B}$ and $\mathrm{C}$ forward information from $\mathrm{A}$, and $\mathrm{C}$ also forwards information to $\mathrm{B}$. The smallest example of a "chamber."

(b)

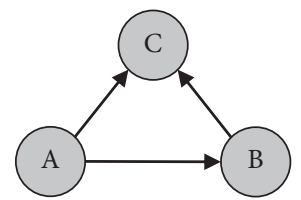

A transitive triad where each participant holds the same opinion -an "echo chamber."

(c)

(d)

FIgURE 3: Structural and attitudinal components of an echo chamber in participation networks.

\section{Communication Patterns in Transport Policy-Making}

4.1. Descriptive Analysis of Network Characteristics of Participation Networks. Descriptive analysis of the network of GCPN and ECPN was firstly calculated with UCINET 6.665 and then visualized through its embedded drawing package NetDraw [49]. To present the key structure in two studied networks, Figure 4 visualizes the simplified networks where marginal nodes with low out-degrees (i.e., have few connections with other network actors) are removed. Different node sizes are proportional to out-degrees of the nodes (i.e., the number of forwarded messages from each participant), and different node shapes are used to distinguish the identity of participants in transport policy networks, with squares indicating organizations/companies, diamonds indicating influential individuals, and circles indicating the general public.

The network of GCPN engages 157 participants who include 49 organizations/companies and 13 influential individuals (see Figure 4(a)), while there is a total of 140 participants and a smaller proportion of verified participants in ECPN, i.e., 24 organizations/companies and 3 influential individuals (see Figure 4(b)). The results show that topics related to bike-sharing business strategies were more attractive to the general public than topics related to government regulations. In other words, in this case, companies can use the Weibo platform to obtain more opinions and feedback from ordinary users from the online communication and participation process. Besides, a similar pattern can be discovered in both networks of GCPN and ECPN in that the entire network was sparse whereas individuals were centralized within several groups (i.e., two major groups in GCPN and three major groups in ECPN). In general, these findings reveal the characteristic of more open and decentralized policy participation than that of traditional topdown policy-making [15].

The network structure statistics were then calculated to quantitively characterize the pattern of networks of GCPN and ECPN (see Table 1). The values of average degree and network density are very close in the two studied networks, which reach 1.02 and $4.43 e-4$ in GCPN and 1.01 and $3.57 e-4$ in ECPN, respectively. It reveals that both of the network participants were weakly connected to others and the majority shared information in a single path. The values of average path length in GCPN and ECPN are around 1. Additionally, the values of network diameter are 4 in GCPN and 5 in ECPN, and the main component fraction reaches approximately $100 \%$ in two networks. The result suggests that the entire participation network on the issue of bikesharing pricing policies was a giant component, and the depth of information dissemination reached around five layers away from the initial messages. It demonstrates the formation of virtual communities that were not limited by geographical and time constraints, and these groups played a significant role in citizen participation and communication in terms of bike-sharing public and private policy-making. Furthermore, the value of the out-degree graph centralization is 0.35 in GCPN and 0.29 in ECPN. We would conclude that there was a tendency for the networks to develop around certain prominent actors, which also can be observed from the network visualization shown in Figure 4. In other words, the power of participants varied rather substantially, and the positional advantages were rather unequally distributed in these two examined networks.

\subsection{Uneven Power and the Different Positions of Participants.}

To investigate the tendency of a substantial amount of concentration in the network of GCPN and ECPN, two indicators were selected to measure the power of each participant (see Figure 5): the number of followers (denoted by $D^{f}$ ), which refers to the cyber power of actors on Weibo; and the number of messages forwarded (out-degree, denoted by $\left.D^{\text {out }}\right)$, which refers to the actors' participation level.

The result shows that the cumulative distributions $D^{f}$ (see Figures 5(a) and 5(c)) and the cumulative out-degree distributions $D^{\text {out }}$ (see Figures $5(\mathrm{~b})$ and $5(\mathrm{~d})$ ) decayed as a 


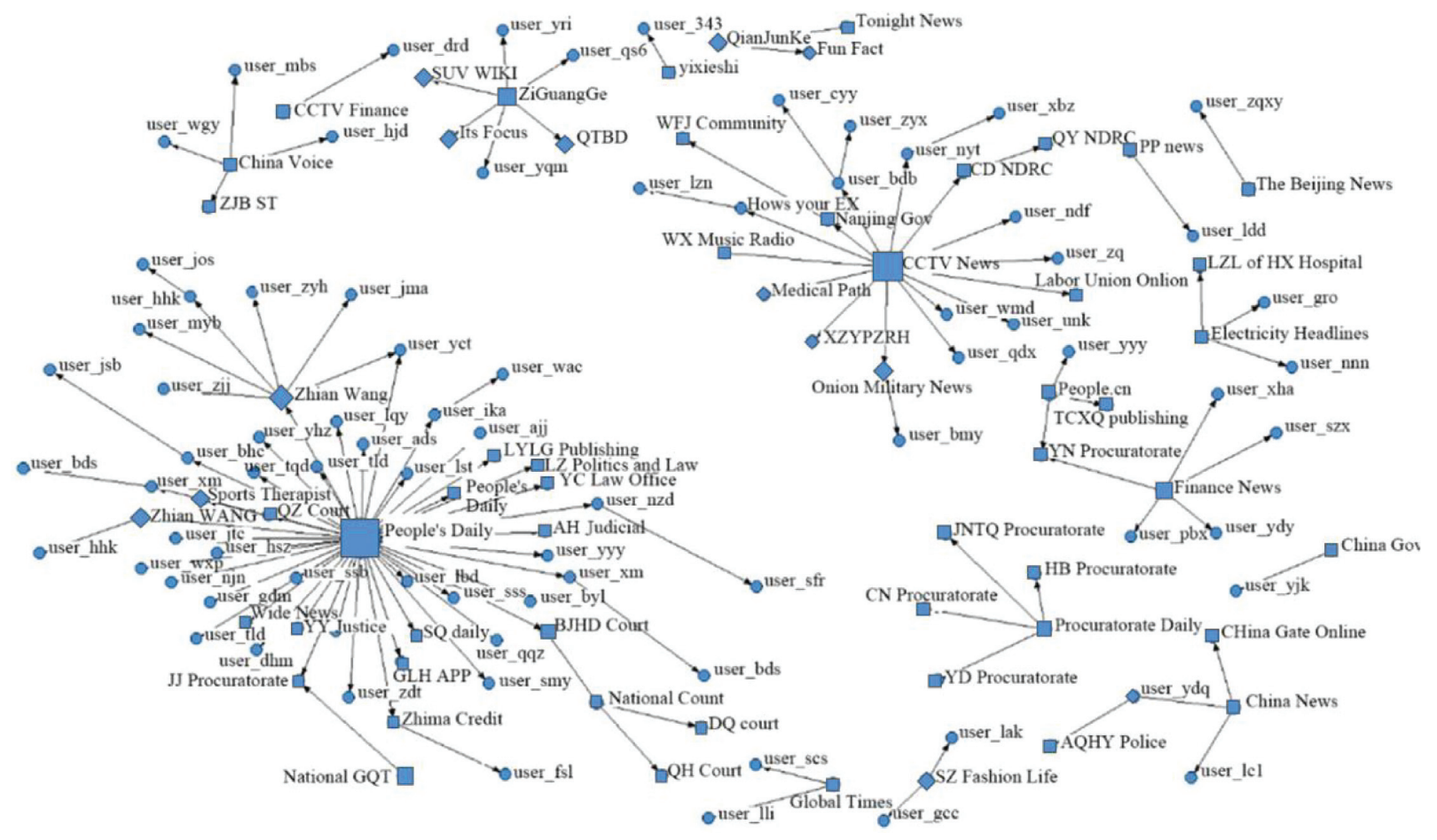

(a)

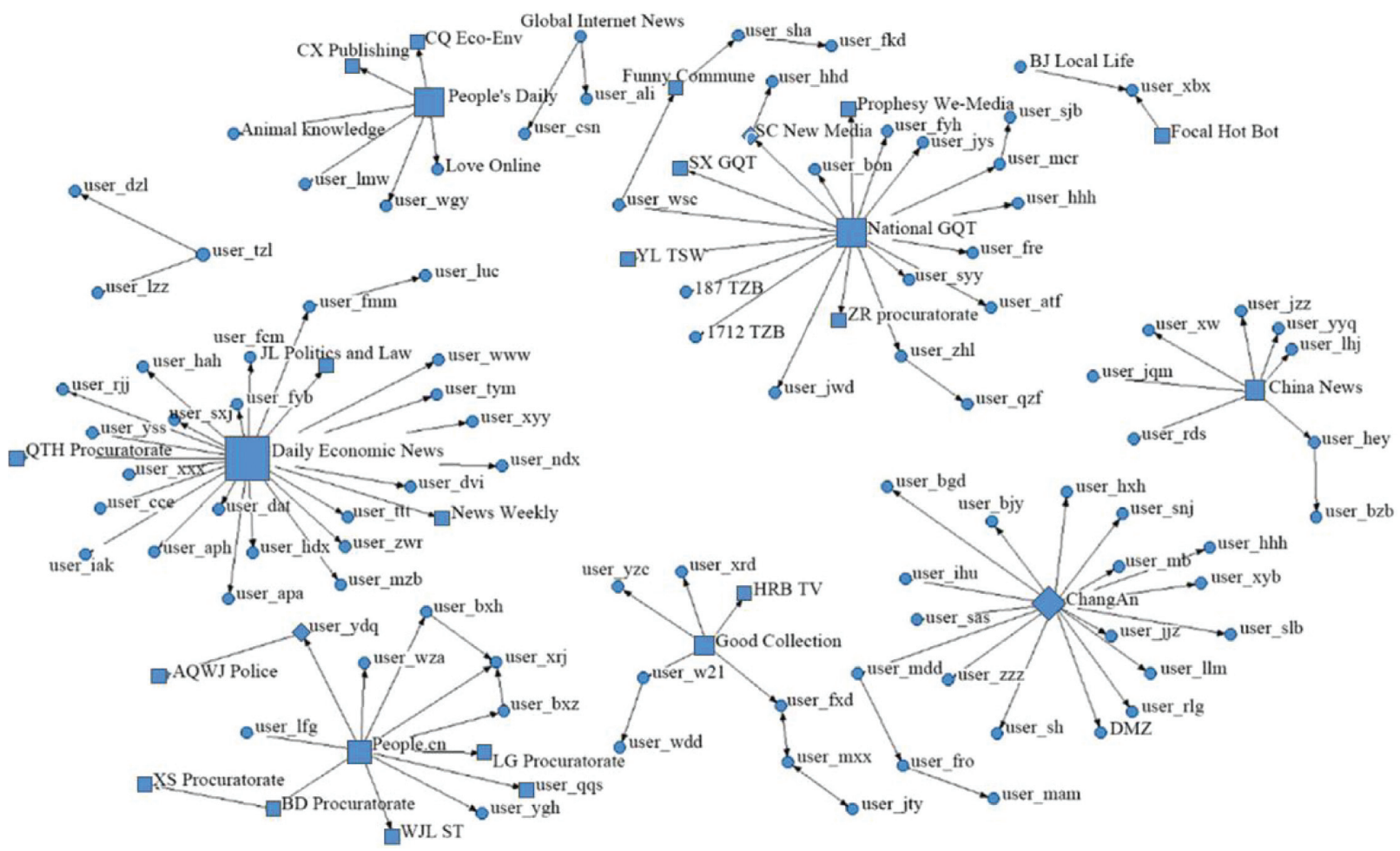

(b)

Figure 4: Simplified network mapping of (a) GCPN and (b) ECPN.

power-law scaling in GCPN and ECPN, suggesting the scalefree characteristic [50]. The pattern of $D^{f}$ indicates that the cyber power of participants was uneven in terms of information diffusion on the topics of bike-sharing pricing policies. The distribution of $D^{\text {out }}$ suggests that the influence of various participants was significantly different because the messages of some actors were forwarded a greater number of times than those of other actors. That is, some participants probably played key roles and influenced others as the "networked power" (prominent participants) [51] in this 
TABLE 1: Statistics of network characteristics.

\begin{tabular}{lcc}
\hline Indicators & GCPN & ECPN \\
\hline Average degree & 1.02 & 1.01 \\
Network density & $4.43 e-4$ & $3.57 e-4$ \\
Average path length & 1.16 & 1.07 \\
Network diameter & 4.00 & 5.00 \\
Main component fraction (\%) & 100.00 & 99.89 \\
Out-degree centralization & 0.35 & 0.29 \\
\hline
\end{tabular}

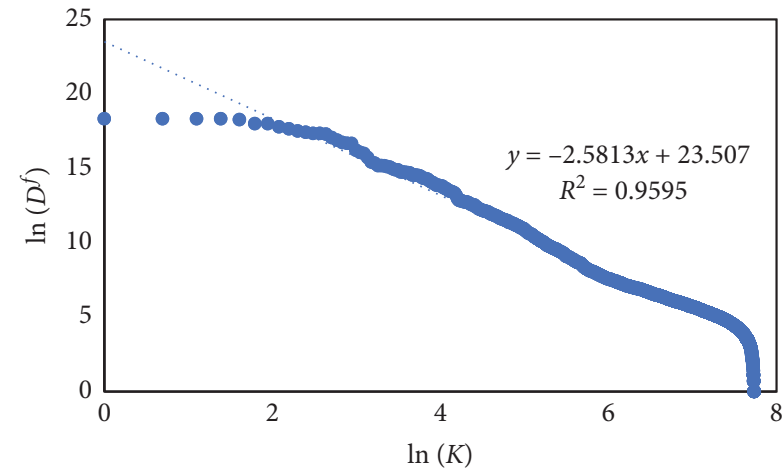

(a)

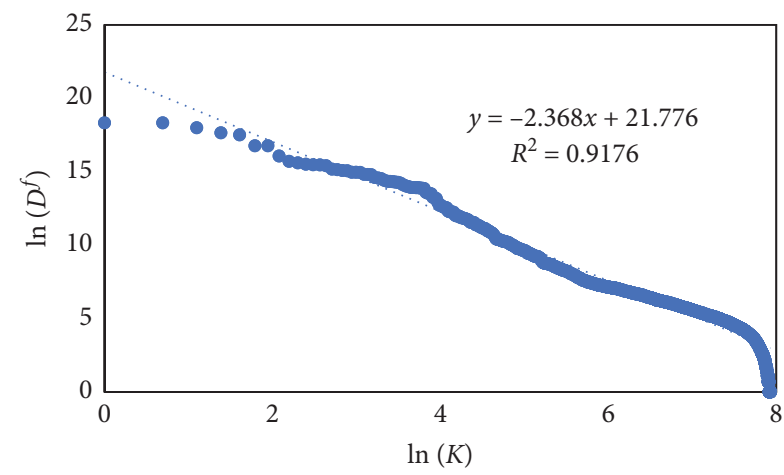

(c)

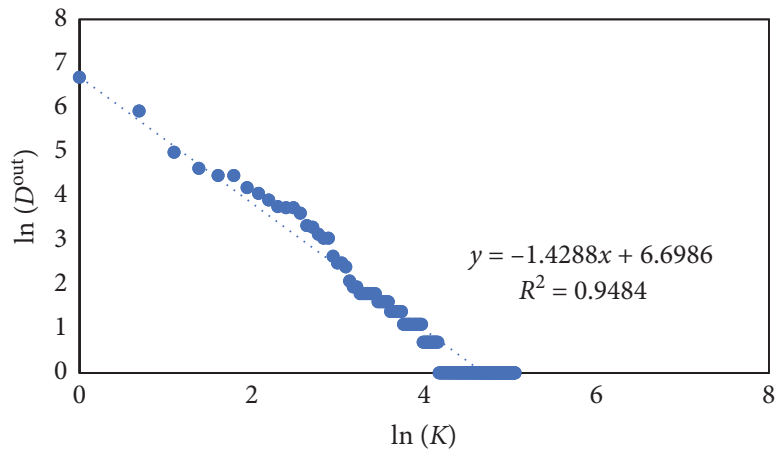

(b)

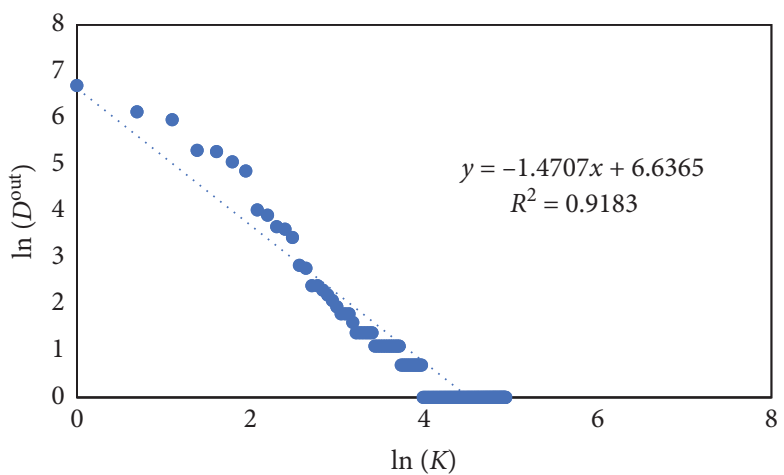

(d)

FIGURE 5: The distribution patterns in log-log graphs: (a) the number of followers $\left(D^{f}\right)$ and (b) the number of messages forwarded (outdegree $\left(D^{\text {out }}\right)$ ) in GCPN, (c) the number of followers $\left(D^{f}\right)$, and $(\mathrm{d})$ the number of messages forwarded (out-degree $\left.\left(D^{\text {out }}\right)\right)$ in ECPN.

case. Taken together, these results provide quantitative evidence on the existence of the unequal power in information sharing of transport policies among various participants in both two studied networks.

\section{Networked Power and Their Roles in Information Dissemination}

5.1. Identifying Power Actors Using Centrality Measurements. To further identify the networked power in forwarding/ forwarded networks of GCPN and ECPN, out-degree centrality and betweenness centrality were calculated. To make the results clear, the 15-top ranked participants in out-DC and $\mathrm{BC}$ lists were selected to analyze their identities and occupational backgrounds (see Figure 6).

In Figure 6(a), organizations/companies and influential individuals occupy the top-15 ranked positions of out-DC in
GCPN and ECPN, while a more proportion of the general public is presented at the top-15 in BC of both networks. This result suggests that verified actors who have the cyber power on Weibo participated actively and were located at the central position (i.e., the "network root") in policy and private policymaking of bike-sharing. In contrast, the result of BC shows that the majority is the general public in this bike-sharing policy network; they played as the "network bridge." The results are shown in Figure 6(b) to help to investigate the background of these influential participants. In terms of "network root," both networks in GCPN and ECPN reveal a similar trend; that is, the majority were media companies and journalists; whereas the "network bridge" in this case were of diverse occupational backgrounds, such as officers from public agencies, academia excepts, and the media.

As the conclusion proposed by Berry [52], it is difficult for views based on a social network to play an important role in 


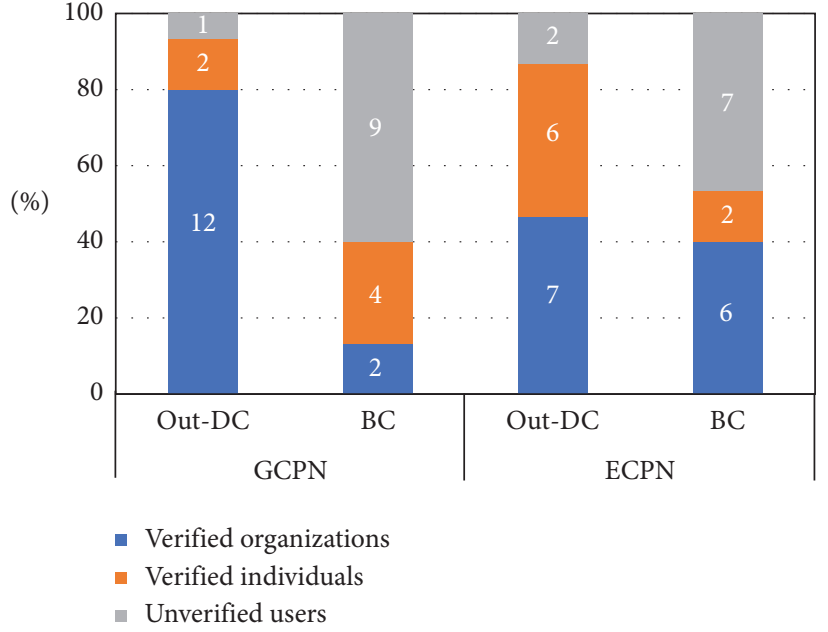

(a)

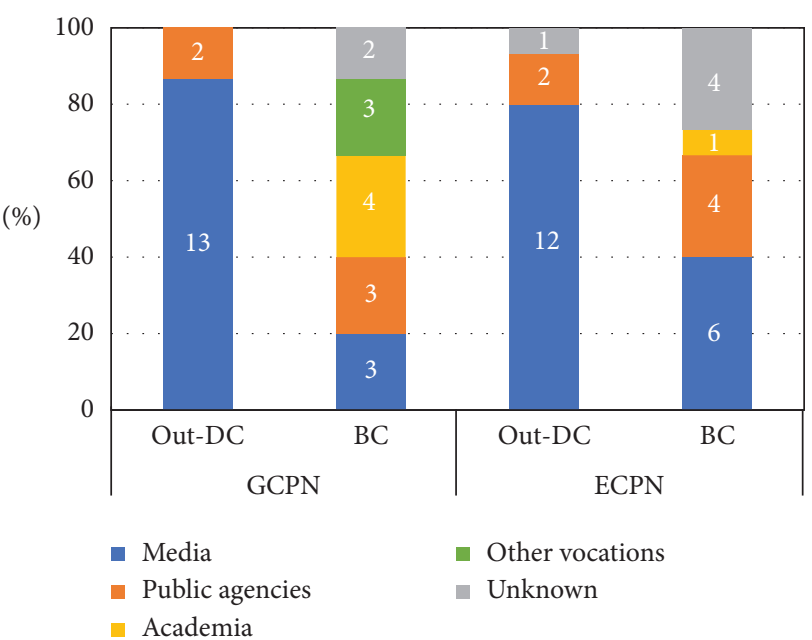

(b)

FIGURE 6: Fifty-top ranked participants aggregated by (a) verification identity and (b) occupational background.

decision-making processes without effective organization and cooperation. In the bike-sharing policy networks, it probably could be explained that organizations/companies and influential individuals established a large number of connections with others in the online discussion, while more diverse social actors were involved to help the information travel further within the virtual community. Therefore, there is a need for transport stakeholders to investigate and understand the needs of people who play different roles and have diverse backgrounds in public and private policy-making.

5.2. Exploring the Hierarchical Structure via K-Core Decomposition. The results of k-core decomposition reveal the hierarchical structure and the subgroups containing influential participants, as visualized in Figure 7, where nodes located at the most peripheral layer (0-shell) are removed. The colors of nodes are used to distinguish the different layers of $\mathrm{k}$-shell, red indicating nodes located in 3-shell and green indicating nodes located in 2-shell. The k-core index of GCPN and ECPN is 3 and 2, respectively. There are 15 nodes in 3shell and 81 nodes in 2-shell of GCPN, while there are 61 nodes in 2-shell of ECPN, which indicates that a small number of participants (i.e., $4.11 \%$ in GCPN and $2.14 \%$ in ECPN) were tightly connected in the networks.

As seen in Figure 7, the network of GCPN presents higher robustness than that of ECPN. The partition of GCPN had a three-layer structure (see Figure 7(a)), its 2-shell was denser with nodes tightly connected in multiple paths, whereas the 2-shell in ECPN is relatively separated or isolated (see Figure 7(b)). The result suggests that the participation network led by governments provided more flexible channels for actors to receive or transfer messages than that led by bike-sharing enterprises. Correspondingly, it could be explained that the aggregated opinions collected from the network of GCPN were closer to the thoughts of the majority on Weibo, while opinions generated from ECPN were probably influenced by their virtual communities.
Concerning the network of GCPN (see Figure 7(b)), there was a community that contains fifty important nodes in the core position. As the statement proposed by Zhao et al. [15], an important characteristic of the social society is that a group or community with similar values can be created and represented a collective identity. To validate the finding in this case, the content of messages and backgrounds related to these fifty participants were further investigated. There were 3 organizations/companies and influential individuals located at the center; the other 12 were the general public; they forwarded messages from three actors without posting any comments. The results reflect the same phenomenon found in Zhao et al.'s study; namely, the participants located in this community probably held the same or similar opinions on public policy-making related to bike-sharing. Another finding is that most of the participants within this 3 -shell structure do not appear at the top-15 list of out-degree centrality and betweenness centrality, which indicates that participants may be of a high $k_{s}$ but a low out-DC or BC. It could be explained that the core-periphery structure is another measurement of the importance of nodes focusing on the community level. Thus, it is suggested to employ various indicators or measurements to evaluate the structure and characteristics of policy networks.

\section{Empirical Evidence of Confirmation Bias in Transport Policy Networks}

6.1. Analysis of the Personal Viewpoints on Weibo. All the messages in two networks of GCPN and ECPN were reviewed and classified into three emotional opinions based on positive, negative, and neutral attitudes. Most participants held one of three viewpoints (see Figure 8):

(i) The majority (over the half) forwarded the messages showing a neutral attitude or without posting any comments, i.e., $62.4 \%$ in GCPN and $55.0 \%$ in ECPN. 


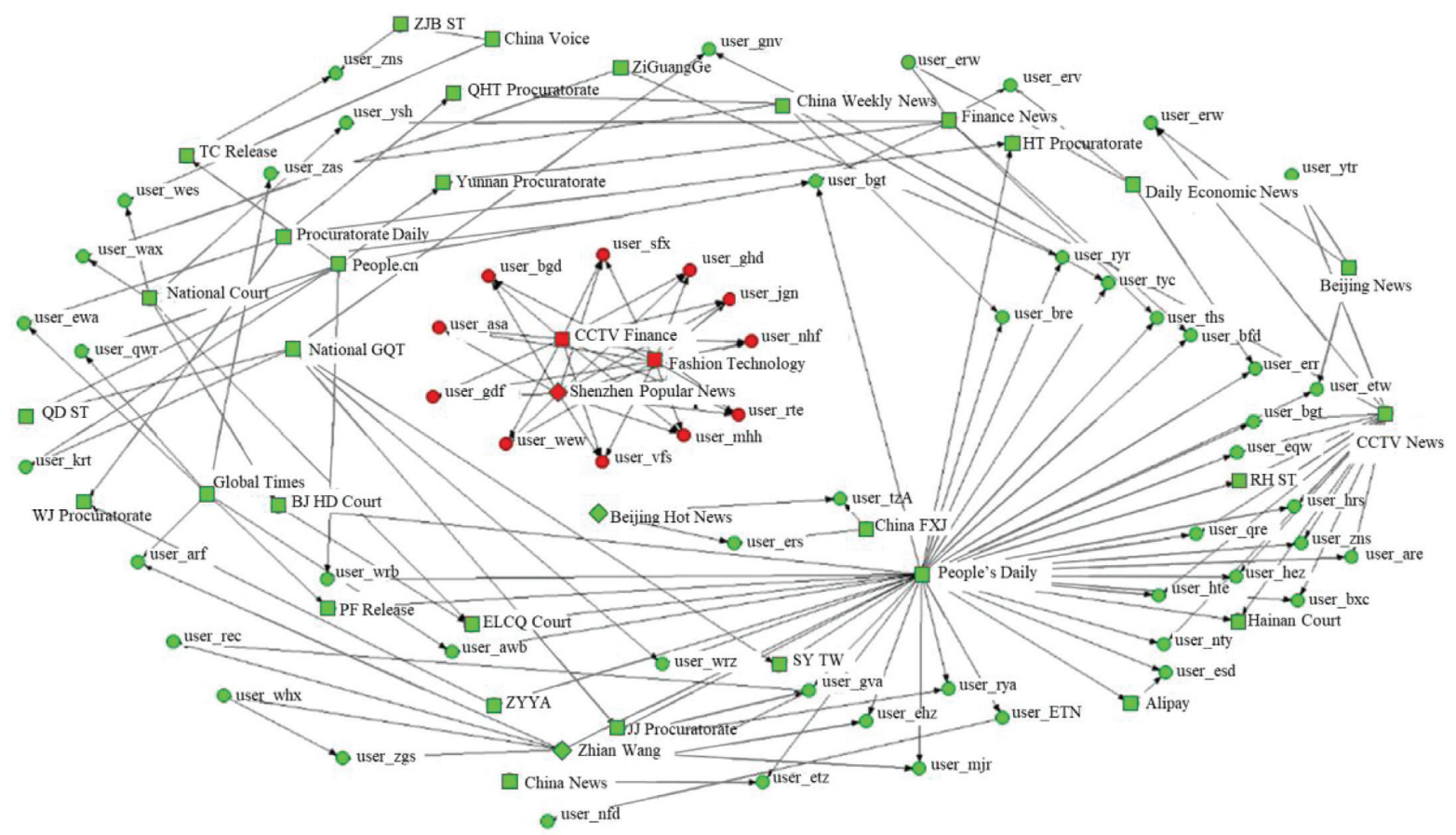

(a)

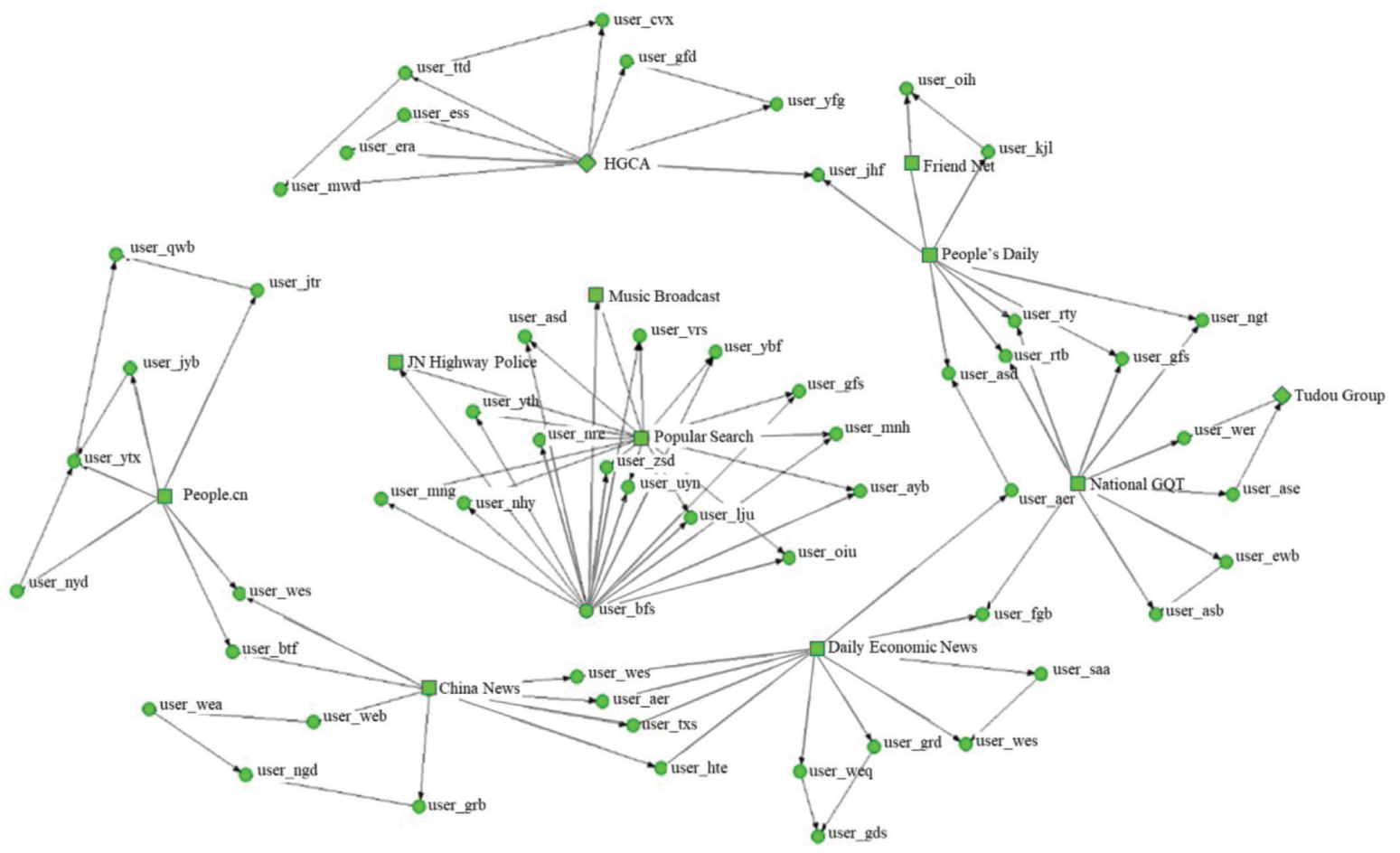

(b)

FIgURE 7: The core-periphery structure measured by K-core decomposition in (a) GCPN and (b) ECPN.

They played a significant role in transmitting their information in the communication process.

(ii) Approximately $20.3 \%$ of the participants in GCPN expressed their pessimistic outlook on the bike-sharing pricing regulations implemented by governments. They worried that whether the policy would be beneficial for refunding due to the dilemma that enterprises faced serious financial problems. At the same time, $33.7 \%$ of the participants in ECPN were against enterprises' increasing prices; they held that it is unreasonable to ride bikes with paying a higher fare that is 


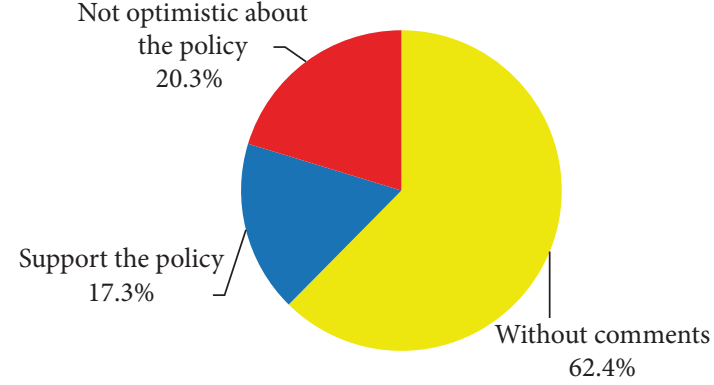

(a)

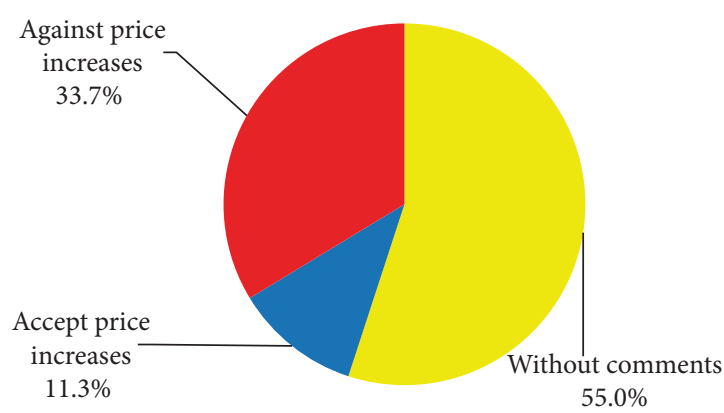

(b)

FIgURe 8: Different opinions in (a) GCPN and (b) ECPN.

even more expensive than that of subway or buses.

(iii) A few of them showed their support or approval of the issue. For example, $17.3 \%$ of participants in GCPN appreciated the efforts paid by governments to regulate the bike-sharing industry and thus protect customers' rights. In ECPN, only $11.3 \%$ of the participants accepted the price increase of bikesharing enterprises.

In general, the distribution of opinions reveals an important characteristic of online public engagement: that is, the majority will not express emotional views on public and private decision-making for major transport issues. The percentage of people holding negative opinions is roughly larger than that of positive opinions in the two examined networks.

6.2. Examination of the Presence of Echo Chambers in Transport Policy Networks. Results corresponding to each step proposed in the identification algorithm are listed in Table 2. It shows that there are five echo chambers and one echo chamber found in GCPN and ECPN, respectively. Generally, the result provides empirical evidence of the existence of echo chambers in participation networks of transport decision-making of public and private sectors.

However, the result shows that the proportion of echo chambers within entire participation networks was quite low, roughly at the same level in GCPN and ECPN (i.e., 3\% and $2 \%$ ). It suggests a relatively low degree of the impact of confirmation bias on online communication toward this bike-sharing case. Another finding is that over half of the chambers (i.e., 63\%) in GCPN were satisfied with the attitudinal components while very few (i.e., 14\%) in ECPN. It could be explained that due to the professional nature of transport governance, the general public is usually limited in participating in the discussion and expression of government policies. Thus, the participants in echo chambers of ECPN were more likely to agree with the views of influential actors, probably by the means of forwarding their messages without comments on social media.

In contrast, the transport service fare is the topic more closely to people's daily life; the business strategy adopted by bike-sharing enterprises attracts more debates among residents. Correspondingly, the echo chamber provided a space needed for viewpoint exchange and free debates on the Internet.

The locations of echo chambers discovered in networks of GCPN and ECPN are visualized in Figure 9. The legend of node colors is changed here to indicate the opinions of each participant, which consists of the legend in Figure 8. All of the echo chambers are distributed in 2-shells; in other words, these participants involved in echo chambers were important actors in the hierarchical structure of transport policy networks as well.

As shown in the figure, there were three echo chambers configured around neutral attitudes (labeled as (i), (ii), and (v)), two echo chambers with negative attitudes (labeled as (iii) and (iv)) in GCPN (see in Figure 9(a)), and one echo chamber configured around neutral attitudes in ECPN (shown in Figure 9(b), labeled as (i)). Additionally, the mechanism of involved participants' identities and the path that echo chambers operate can be observed in the figure. In Figure 9(a), the echo chamber of neutral attitudes in GCPN existed in the information flow between organizations/companies (e.g., court departments) or from organizations/companies (e.g., nationwide news media) to the general public, whereas the echo chamber of negative opinions was presented in the message diffusion from influential individuals (e.g., famous journalists) to the general public. In contrast, as seen in Figure 9(b), an echo chamber with neutral attitudes was configured around an organization/ company (e.g., nationwide news media) and two general participants. Furthermore, there is a phenomenon that happened in GCPN; namely, echo chambers in policy networks of this case were overlapped among participants in two echo chambers; see examples with labeling (i) and (ii) in Figure 9(a).

The above results could be explained; the networked power with greater influence and readership on social media was probably becoming the actors that echo/amplify others' viewpoints and understanding of major transport issues. Furthermore, according to this case, echo chambers were more likely to be configured around participants with pessimistic or neutral attitudes on bike-sharing pricing policies. 
TABLE 2: The summary of echo chamber identification in participation networks.

\begin{tabular}{lcc}
\hline Count & GCPN & ECPN \\
\hline Step 1: multiforward participants & 75.00 & 57.00 \\
Step 2: like-shaped transitive triads & 10.00 & 20.00 \\
Step 3: chamber structure & 8.00 & 7.00 \\
Step 4: echo chambers & 5.00 & 1.00 \\
Percentage (\%) & GCPN & ECPN \\
Echo chambers within networks & 0.03 & 0.02 \\
Echo chambers within chambers & 0.63 & 0.14 \\
\hline
\end{tabular}

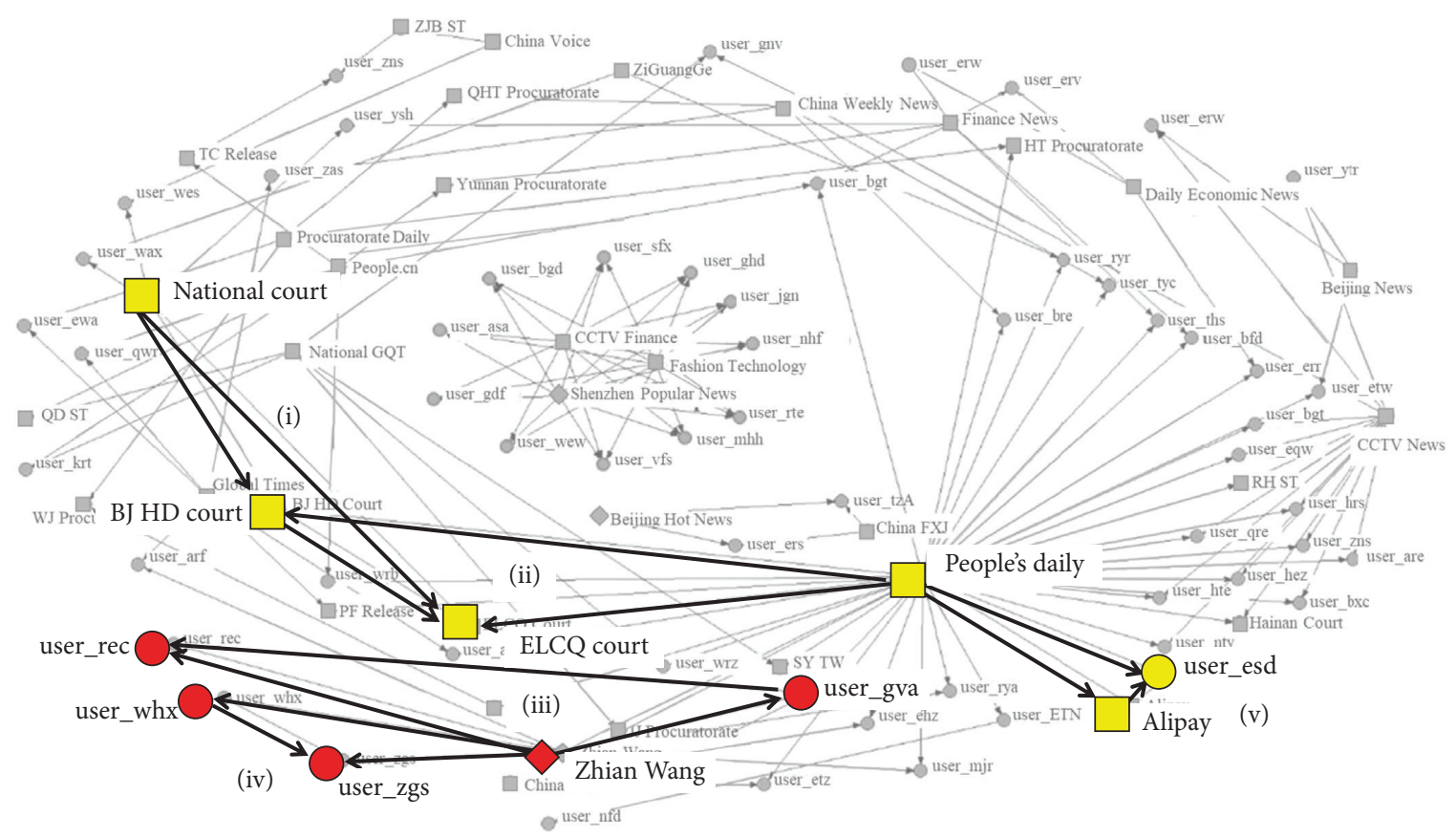

(a)

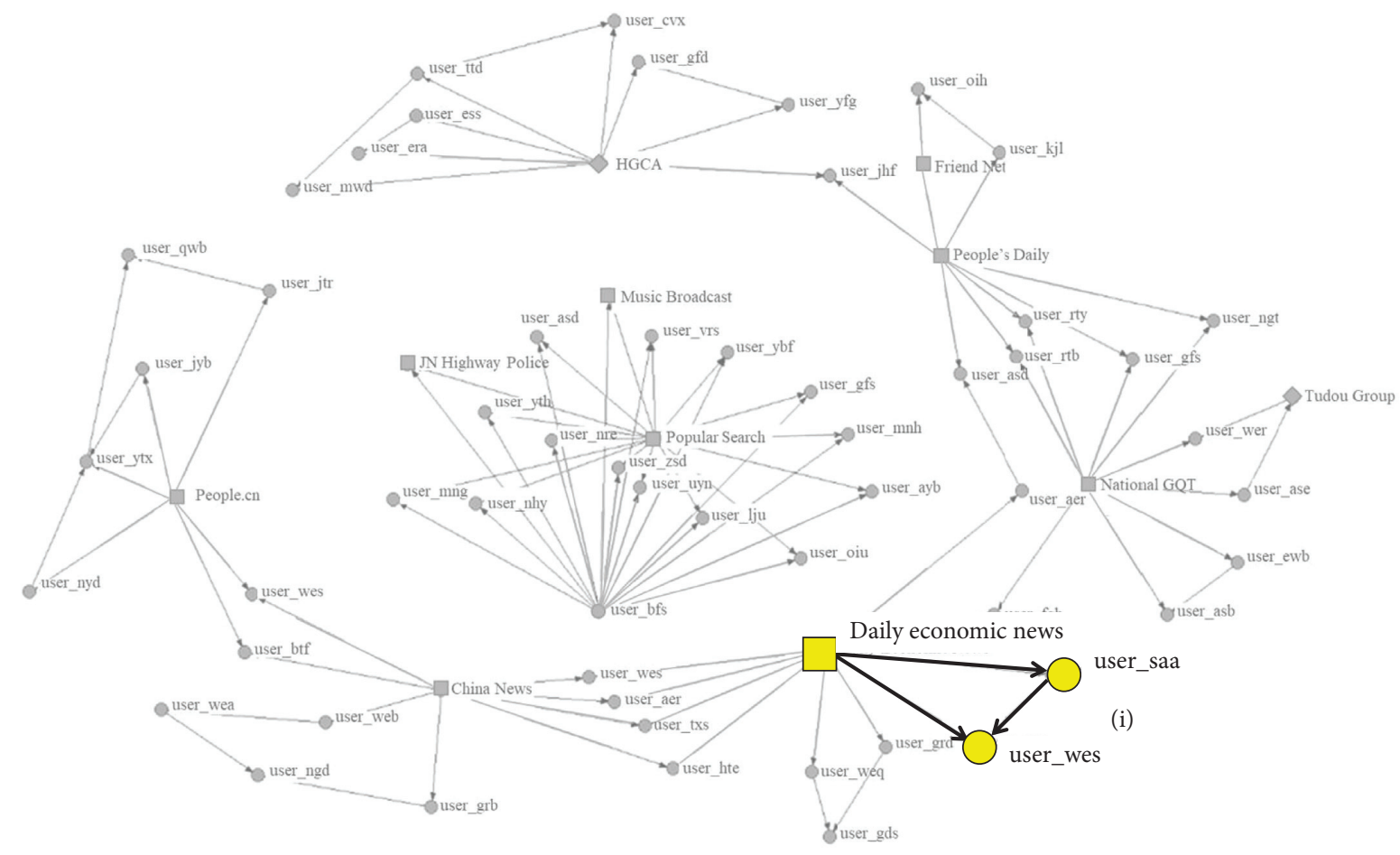

(b)

FIgURE 9: Illustration of the existence of echo chambers in the structure of (a) GCPN and (b) ECPN. 


\section{Conclusion and Discussion}

This study aims to qualitatively characterize the patterns of two participation networks in public and private policymaking and further empirically examines the presence of echo chambers in terms of information dissemination. Besides, the study provides a new network-based analytical framework focusing on the behavioral logic in the exploration and the collaborative interactions among multistakeholders related to transport policies. To summarize, the main conclusions are remarks from the findings that are listed as follows:

(i) The descriptive analysis results provide evidence that multiple actors, especially the general public, share information in GCPN and ECPN. However, the participation networks are sparse and affected by a small component of participants with greater cyber influence and do not cover all crucial roles than others in information diffusion.

(ii) The results suggest that when characterizing the key actors in information diffusion of both networks, organizations/companies and influential individuals (i.e., media companies, and journalists) contribute more actively as the "network root" to be at the central position, whereas the general public coming from various occupational backgrounds are more prominent as the "network bridge" to transfer the information further.

(iii) Through the k-core decomposition method, the two studied networks exhibit the core-periphery structure as a small number of participants build a community with similar views but different backgrounds.

(iv) The opinion analysis result reveals that the majority of participants will not express emotional viewpoints in transport policies. Furthermore, there is a general tendency that people are more likely to hold negative attitudes on social media.

(v) The empirical results demonstrate the existence of echo chambers in both GCPN and ECPN, although its prevalence proportion is relatively low. Besides, echo chambers are configured around participants with critical opinions related to transport policies, and they generally happened such that organizations or influential individuals echoed the views of the general public.

These findings also have several managerial and policy implications. First, this study demonstrates the potential of using social media as a channel to investigate public participation among multi-stakeholders on major transport issues. Various measurements and indicators (e.g., network centrality, core-periphery) from the network perspective can be applied to analyze the relationship among actors. Second, it emphasizes the possibility of confirmation bias in transport policy networks that might be caused by the multistakeholders with diverse interests and their complex interactions. Policymakers and service providers should collect a more complete sample of people's views or consider diverse platforms in public engagement. Third, once the impact of confirmation bias is confirmed, it is suggested to conduct a further exploration based on the content of collected samples to improve the reliability of the result, such as topic models and sentiment analysis.

As a presentative of emerging mobility services, the service providers of bike-sharing are driven by complex interests, including being more likely to obtain a large number of user traffic instead of promoting travel services in the short term, gaining a higher market share to increase their valuation in the risk market, etc. Besides, its major users have certain characteristics, including being younger and willing to accept emerging techniques. Therefore, when using social media as a platform of public participation, bike-sharing policies may have a different/biased communication and participation process which is different from other urban transport policies. With the emergence of more new mobility services, the stakeholders may be more motivated and capable of intervening in transport policies. This is a topic that deserved continuous attention in the effective collaborative governance of urban transport. Empirical studies on a wider range of cases are needed to obtain a more profound understanding.

Despite its contributions and practical implications, this study still has several limitations. Its analysis is restricted to a specific participation network for bike-sharing price strategies, which may limit the generalizability of the empirical results to urban transport, other policy, and cultural contexts (e.g., different affecting or affected interest groups, the degree of willingness to express opinions through Internet, and participation channels available to the public). Further cross-disciplinary and cross-cultural research studies could be conducted to validate or expand the findings presented here. Furthermore, limited by the focus of revealing the key pattern of participation networks, this study only analyzes the forwarding/forwarded relationship represented by the single-directed, unweighted network. Further studies could include complete interaction relationships and features among participants included in the analysis.

\section{Data Availability}

The Weibo data used to support the findings of this study have not been made available because of the privacy protection rules of users' data.

\section{Conflicts of Interest}

The authors declare that there are no conflicts of interest regarding the publication of this paper.

\section{Acknowledgments}

The authors acknowledge support from the project of the National Natural Science Foundation of China (Nos. 71734004 and 71603186), Shanghai Pujiang Program (No. 2019PJC109), and China Scholarship Council (No. 201906260112). It was also partially supported by C2SMART 
Tier 1 University Transportation Center at New York University.

\section{References}

[1] L. Zhou, J. Ye, and X. Chen, Current Situation and Problem Analysis of Public Participation in China's Urban Transportation Planning, Transportation Research Board, Washington, DC, USA, 2015.

[2] J. Gonzalez-Feliu, C. Pronello, and J. M. S. Grau, "Multistakeholder collaboration in urban transport: state-of-the-art and research opportunities," Transport, vol. 33, no. 4, pp. 1079-1094, 2018.

[3] A. Alfayez, Z. Awwad, C. Kerr et al., "Understanding gendered spaces using social media data," in Social Computing and Social Media: Applications and Analytics, Scsm 2017, Pt II, G. Meiselwitz, Ed., vol. 10283, pp. 338-356, Springer, Cham, Switzerland, 2017.

[4] L. Mandarano, M. Meenar, and C. Steins, "Building social capital in the digital age of civic engagement," Journal of Planning Literature, vol. 25, no. 2, pp. 123-135, 2010.

[5] A. Gal-Tzur, S. M. Grant-Muller, E. Minkov, and S. Nocera, "The impact of social media usage on transport policy: issues, challenges and recommendations," Edited by J. F. DeSousa, J. P. DeSousa, A. Costa, T. Farias, and S. Melo, Eds., in Transportation: Can We Do More with Less Resources?-16th Meeting of the Euro Working Group on Transportation-Porto 2013, vol. 111, pp. 937-946, 2014.

[6] S. Plous, The Psychology of Judgment and Decision Making, Mcgraw-Hill Book Company, New York, NY, USA, 1993.

[7] E. Pariser, The Filter Bubble: How the New Personalized Web Is Changing what We Read and How We Think, Penguin, London, UK, 2011.

[8] M. Salerno, T. Sanchez, S. Tomasello, and T. Metz, Practices for Online Public Involvement (Project 20-05, Topic 49-11), Transportation Research Board, Washington, DC, USA, 2019, http://www.trb.org/Publications/Blurbs/179304.aspx.

[9] A. Gal-Tzur, S. M. Grant-Muller, T. Kuflik, E. Minkov, S. Nocera, and I. Shoor, "The potential of social media in delivering transport policy goals," Transport Policy, vol. 32, pp. 115-123, 2014.

[10] Y. Cheng, "Collaborative planning in the network: consensus seeking in urban planning issues on the Internet-the case of China," Planning Theory, vol. 12, no. 4, pp. 351-368, 2013.

[11] S. M. Kaufman, How Social Media Moves New York: Twitter Use by Transportation Providers in the New York Region, Transportation Research Board, Washington, DC, USA, 2012, http://wagner.nyu.edu/files/faculty/publications/how_social_ media_moves_new_york.pdf.

[12] B. Batrinca and P. C. Treleaven, "Social media analytics: a survey of techniques, tools and platforms," Ai \& Society, vol. 30, no. 1, pp. 89-116, 2015.

[13] C. Collins, S. Hasan, and S. V. Ukkusuri, “A novel transit rider satisfaction metric: rider sentiments measured from online social media data," Journal of Public Transportation, vol. 16, no. 2, p. 2, 2013.

[14] L. Tang, Z. Duan, and Y. Zhao, "Toward using social media to support ridesharing services: challenges and opportunities," Transportation Planning and Technology, vol. 42, no. 4, pp. 355-379, 2019.

[15] M. Zhao, Y. Lin, and B. Derudder, "Demonstration of public participation and communication through social media in the network society within Shanghai," Environment and Planning B: Urban Analytics and City Science, vol. 45, no. 3, pp. 529-547, 2017.
[16] B. A. Furtado, M. A. Fuentes, and C. J. Tessone, "Policy modeling and applications: state-of-the-art and perspectives," Complexity, vol. 2019, Article ID 5041681, 11 pages, 2019.

[17] S. Wasserman and K. Faust, Social Network Analysis: Methods and Applications, Vol. 8, Cambridge University Press, Cambridge, UK, 1994.

[18] J. Scott, Social Network Analysis: A Handbook, Sage publications, London, UK, 2nd edition, 2000.

[19] L. Xie, T. Han, and M. Skitmore, "Governance of relationship risks in megaprojects: a social network analysis," Advances in Civil Engineering, vol. 2019, Article ID 1426139, 13 pages, 2019.

[20] Y. Tang, G. Wang, H. Li, D. Cao, and X. Li, "Comparing project-based collaborative networks for BIM implementation in public and private sectors: a longitudinal study in Hong Kong," Advances in Civil Engineering, vol. 2019, Article ID 6213694, 14 pages, 2019.

[21] D. P. Cheung and M. H. Gunes, "A complex network analysis of the United States air transportation," in Proceedings of the 2012 IEEE/ACM International Conference on Advances in Social Networks Analysis and Mining, Istanbul, Turkey, August 2012.

[22] M. G. Song and G. T. Yeo, "Analysis of the air transport network characteristics of major airports," The Asian Journal of Shipping and Logistics, vol. 33, no. 3, pp. 117-125, 2017.

[23] S. Dörry and A. Decoville, "Governance and transportation policy networks in the cross-border metropolitan region of Luxembourg: a social network analysis," European Urban and Regional Studies, vol. 23, no. 1, pp. 69-85, 2013.

[24] H. Sun, Y. Zhang, Y. Wang, L. Li, and Y. Sheng, "A social stakeholder support assessment of low-carbon transport policy based on multi-actor multi-criteria analysis: the case of Tianjin," Transport Policy, vol. 41, pp. 103-116, 2015.

[25] K. Weiss, M. Hamann, M. Kinney, and H. Marsh, "Knowledge exchange and policy influence in a marine resource governance network," Global Environmental Change, vol. 22, no. 1, pp. 178-188, 2012.

[26] M. Mills, J. G. Álvarez-Romero, K. Vance-Borland et al., "Linking regional planning and local action: towards using social network analysis in systematic conservation planning," Biological Conservation, vol. 169, pp. 6-13, 2014.

[27] R. S. Nickerson, "Confirmation bias: a ubiquitous phenomenon in many guises," Review of General Psychology, vol. 2, no. 2, pp. 175-220, 1998.

[28] P. M. Wagner and T. Ylä-Anttila, "Can policy forums overcome echo chamber effects by enabling policy learning? Evidence from the Irish climate change policy network," Journal of Public Policy, vol. 40, no. 2, pp. 194-211, 2018.

[29] K. Garimella, G. De Francisci Morales, A. Gionis, and M. Mathioudakis, "The effect of collective attention on controversial debates on social media," in Proceedings of the 2017 ACM, pp. 43-52, Troy, MI, USA, June 2017.

[30] M. Del Vicario, G. Vivaldo, A. Bessi et al., "Echo chambers: emotional contagion and group polarization on Facebook," Scientific Reports, vol. 6, no. 1, p. 37825, 2016.

[31] M. Cinelli, G. D. F. Morales, A. Galeazzi, W. Quattrociocchi, and M. Starnini, "Echo chambers on social media: a comparative analysis," 2020, https://arxiv.org/abs/2004.09603.

[32] A. Bessi, M. Coletto, G. A. Davidescu, A. Scala, G. Caldarelli, and W. Quattrociocchi, "Science vs conspiracy: collective narratives in the age of misinformation," PLoS One, vol. 10, no. 2, Article ID e0118093, 2015.

[33] A. Cossard, G. D. F. Morales, K. Kalimeri et al., "Falling into the echo chamber: the italian vaccination debate on Twitter," 2020, https://arxiv.org/abs/2003.11906. 
[34] C. R. Sustein, Republic. Com 2.0, Princeton University Press, Princeton, NJ, USA, 2009.

[35] "Facebook's CEO Mark Zuckerberg's open letter," 2017, https://www.facebook.com/notes/mark-zuckerberg/buildingglobal-community/10103508221158471/.

[36] L. Jasny, J. Waggle, and D. R. Fisher, "An empirical examination of echo chambers in US climate policy networks," Nature Climate Change, vol. 5, no. 8, pp. 782-786, 2015.

[37] E. Colleoni, A. Rozza, and A. Arvidsson, "Echo chamber or public sphere? Predicting political orientation and measuring political homophily in Twitter using big data," Journal of Communication, vol. 64, no. 2, pp. 317-332, 2014.

[38] L. Jasny, A. M. Dewey, A. G. Robertson et al., "Shifting echo chambers in US climate policy networks," PLoS One, vol. 13, no. 9, Article ID e0203463, 2018.

[39] F. Ma, W. Shi, K. F. Yuen, Q. Sun, and Y. Guo, "Multistakeholders' assessment of bike sharing service quality based on DEMATEL-VIKOR method," International Journal of Logistics Research and Applications, vol. 22, no. 5, pp. 449472, 2019.

[40] Ofo Faces Flak on Refunds of Deposits, China Daily, Beijing, China, 2018, https://www.chinadaily.com.cn/a/201812/20/ WS5c1ad088a3107d4c3a001bde.html.

[41] Tighter Controls on Transport Deposits, China Daily, 2019, http://global.chinadaily.com.cn/a/201905/17/ WS5cde0cdfa3104842260bc30b.html.

[42] C. Noy, "Sampling knowledge: the hermeneutics of snowball sampling in qualitative research," International Journal of Social Research Methodology, vol. 11, no. 4, pp. 327-344, 2008.

[43] R. A. Hanneman and M. Riddle, "A brief introduction to analyzing social network data," The Sage Handbook of Social Network Analysis, pp. 331-339, SAGE Publications Ltd, Thousand Oaks, CA, USA, 2011.

[44] A. Punel and A. Ermagun, "Using Twitter network to detect market segments in the airline industry," Journal of Air Transport Management, vol. 73, pp. 67-76, 2018.

[45] M. Newman, "Power laws, Pareto distributions and Zipfs law," Contemporary Physics, vol. 46, no. 5, pp. 323-351, 2005.

[46] L. C. Freeman, "Centrality in social networks conceptual clarification," Social Networks, vol. 1, no. 3, pp. 215-239, 1978.

[47] L. C. Freeman, "A set of measures of centrality based on betweenness," Sociometry, vol. 40, no. 1, pp. 35-41, 1977.

[48] I. Alvarez-Hamelin, L. Dall'Asta, A. Barrat, and A. Vespignani, "k-core decomposition: a tool for the visualization of large scale networks," 2005, https://arxiv.org/abs/ cs/0504107.

[49] S. P. Borgatti, M. G. Everett, and L. C. Freeman, Ucinet for Windows: Software for Social Network Analysis, Analytic Technologies, Harvard, MA, USA, 2002.

[50] A.-L. Barabási and R. Albert, "Emergence of scaling in random networks," Science, vol. 286, no. 5439, pp. 509-512, 1999.

[51] M. Castells, "Network theory-A network theory of power," International Journal of Communication, vol. 5, p. 15, 2011.

[52] L. L. Berry, "The collaborative organization:," Organizational Dynamics, vol. 33, no. 3, pp. 228-242, 2004. 